\title{
THE GLAST GUEST INVESTIGATOR PROGRAM
}

\author{
David L. Band ${ }^{*, \dagger}$ and the GLAST SSC* \\ ${ }^{*}$ Code 661, NASA/GSFC, Greenbelt, MD 20771 \\ ${ }^{\dagger}$ CRESST/UMBC
}

\begin{abstract}
We provide an overview of the GLAST Guest Investigator (GI) program, which will support basic research relevant to the GLAST mission in yearly cycles beginning approximately two months after launch. Current details about the GLAST GI program will always be posted on the GLAST Science Support Center (GSSC) website: http://glast.gsfc.nasa.gov/ssc/.
\end{abstract}

Keywords: Gamma-Ray Missions

PACS: $95.55 . \mathrm{Ka}$; 95.85.Pw; $98.70 . \mathrm{Rz}$

\section{INTRODUCTION}

The GLAST Guest Investigator (GI) program will support the scientife community's participation in the GLAST mission by funding GLAST-related data analysis and research, by providing simultaneous observations through joint observing programs with other astronomical facilities, and by modifying GLAST's observing program. Because the GI program will change from year to year, here we provide an overview of the less ephemeral aspects of the program. The GLAST Science Support Center (GSSC) is the mission's interface with the scientifc community, and the GSSC website (http://glast.gsfc.nasa.gov/ssc/) will always provide specifcs regarding the GI program.

\section{THE GLAST DATA AND THE DATA RELEASE POLICY}

To understand the opportunities afforded by the GI program, the nature of the GLAST data and the policies guiding its release must be considered. The LAT is a wide feld-of-view (FOV) detector (most analyses will include data up to $65^{\circ}$ off-axis) that will usually scan the sky. In addition the relatively large (compared to other wavelength bands) point-spread function $\left(<3.5^{\circ}\right.$ at $100 \mathrm{MeV},<0.15^{\circ}$ at $\left.>10 \mathrm{GeV}\right)$ will require the simultaneous analysis of all the sources in a region $\sim 10-15^{\circ}$ around the source of interest. Because the LAT will usually accumulate data on a large fraction $(\sim 30 \%)$ of the sky at any time, and the scan pattern will provide uniform sky coverage on $\sim 3$ hour timescales, pointed observations will rarely be scientifcally warranted: the more rapid accumulation of exposure for one source will disrupt the exposure accumulation for sources over the rest of the sky. Therefore, the LAT event data will not easily be broken into observations of individual sources, as is done for narrow FOV instruments.

Consequently, the general policy is that the GLAST data will be released publicly as soon as they have been processed by the instrument teams and loaded into the GSSC's databases (a latency of less than two days). However, during the Erst year of scientifc operations, which coincides with the GI program's frst cycle, the LAT instrument team will be calibrating the LAT, fne-tuning the analysis software, and preparing a point source to guide future analysis. Therefore, during this frst year LAT event data will be proprietary to the LAT instrument team. During this period the LAT team will make public auxes, spectra and lightcurves of detected transients and $\sim 20$ selected sources (the list is posted on the GSSC website). At the end of the frst year, all Cycle 1 and subsequent LAT event data will become publicly available. GBM science data will be released from the beginning of science operations.

Thus in general GIs may propose to analyze all GLAST scientifc data without a proprietary period. However during Cycle 1 GIs may not propose to analyze LAT event data, but may propose to study all other released data.

\section{GUEST INVESTIGATOR PROGRAM BASICS}

The annual 'cycles' of the GLAST GI Program will be announced in NASAŠs Research Opportunities in Space and Earth Science (ROSES); for example, Cycle 1 was announced in the 2007 ROSES. This announcement is the defnitive, 
legal defnition of the program. The GSSC will administer this program for NASA Headquarters.

Investigators may propose the following investigation types:

- The analysis of released LAT scientifc data. Note that during Cycle 1 this includes duxes, spectra and lightcurves of transients and a list of $\sim 20$ interesting sources, as well as a preliminary source list that will be released half-way through the cycle. After the frst year all LAT event data will be public without a proprietary period.

- The analysis of burst data from the GBM.

- Multiwavelength observations with other instruments and observatories that are directly relevant to GLAST science objectives. Observations on other astronomical facilities may be awarded through joint programs between GLAST and these facilities.

- Development of data analysis techniques applicable to the data provided by the GLAST instruments.

- Theoretical investigations that will advance the mission science return of GLAST. Up to 10\% of GLAST GI funding may be devoted to such theoretical efforts.

- Changes in the observing plan (e.g., pointed observations), after the \&rst year. However, most scientifc objectives will be met by the default survey mode. Note that in Cycle 1 GI proposals cannot modify the observing plan.

Further information can be found on the GSSC website.

There will be two proposal classes. Regular proposals will provide research plans that can be completed in one year. Large proposals will have research plans that are more expansive and may take up to three years to complete. The number of large projects funded, and corresponding budgets awarded in any given year, will be limited. Large projects will be reviewed each year to determine if appropriate progress is being made. PIs of approved large projects must submit a annual progress report.

The Cycle 1 program will (tentatively) fund $\sim 40-50$ regular and $\sim 4$ large proposals. The GI program in subsequent years will be approximately twice as large.

PIs at institutions outside the US may submit proposals, but cannot be funded by NASA (positive evaluations may be useful for obtaining funding from non-US funding agencies). US Co-Is of proposals submitted by foreign PIs may be funded commensurate with their level of effort.

The GI program will include a GLAST Fellows Program with a schedule similar to the Hubble and Chandra Fellows programs.

More information available from GSSC website: http://glast.gsfc.nasa.gov/ssc/ Assistance is available from the GSSC helpdesk: http://glast.gsfc.nasa.gov/ssc/help/.

\section{PROPOSAL SUBMISSION}

A two stage proposal process will be used in Cycle 1, and is planned for subsequent cycles. First, proposers will submit their 4 page science proposals that will be evaluated by peer panels. Second, those GIs whose scientifc proposals are tentatively selected will then submit the proposalšs funding portion. Proposers will submit the maximum funding request (a single number) with the science proposals, but will submit a detailed budget only if the science proposal has been tentatively accepted. For Cycle 1 the science proposals will be submitted through the Remote Proposal System (RPS) at Goddard Space Flight Center. If the science proposal has been tentatively accepted, the budgets will be submitted through the NASA Solicitation and Proposal Integrated Review and Evaluation System (NSPIRES).

To assist scientists prepare GI proposals, the GSSC will provide methods to estimate the detectability of LAT point sources (e.g., the time required for the $5 \sigma$ detection of a source). The GSSC will also provide a GLAST version of WebSpec, a web-based tool that simulates spectra by running XSPECŠs fakeit command; thus scientists can simulate and $£ t$ GBM or LAT spectra. A technical handbook will be posted on the GSSC website.

In the second and subsequent cycles scientists will be able to simulate data using the tools in the Science Analysis Environment (SAE), the set of tools that will be provided to analyze GLAST data. A spatial-spectral source model can be folded through the LAT instrument response functions to simulate an observation. The simulated data can then be analyzed with the SAE tools. Because during the frst cycle LAT count data will not be released and GIs cannot propose observations, the relevant $\mathrm{SAE}$ tools will not be available for the Erst round of proposals. 